\title{
Backbone cup - a structure design competition based on topology optimization and 3D printing
}

\author{
Ji-Hong Zhu*, Kai-Ke Yang, and Wei-Hong Zhang \\ Engineering Simulation and Aerospace Computing (ESAC), Northwestern Polytechnical University, Xi'an, Shaanxi 710072, P.R. China
}

Received 12 January 2016 / Accepted 17 January 2016

\begin{abstract}
This paper addresses a structure design competition based on topology optimization and 3D Printing, and proposes an experimental approach to efficiently and quickly measure the mechanical performance of the structures designed using topology optimization. Since the topology optimized structure designs are prone to be geometrically complex, it is extremely inconvenient to fabricate these designs with traditional machining. In this study, we not only fabricated the topology optimized structure designs using one kind of 3D Printing technology known as stereolithography (SLA), but also tested the mechanical performance of the produced prototype parts. The finite element method is used to analyze the structure responses, and the consistent results of the numerical simulations and structure experiments prove the validity of this new structure testing approach. This new approach will not only provide a rapid access to topology optimized structure designs verifying, but also cut the turnaround time of structure design significantly.
\end{abstract}

Key words: Structure design competition, Topology optimization, Stereolithography, Quick structure testing.

\section{Introduction}

Ever since the first time Bendsøe [1], Suzuki and Kikuchi [2] used homogenization based approach to solve structural topology optimization problems, many scholars had focused their attention on this appealing filed of structure optimization. The latter researches proposed the solid isotropic material with penalization (SIMP) method [3-5] which made it possible to gain practical structure design through topology optimization, the ESO method [6], and the level set method [7], etc. For recent advances of topology optimization, the readers are referred to the literature surveys of Deaton and Grandhi [8], Guo and Cheng [9]. Meanwhile, structural topology optimization has been used widely in automobiles [10], buildings [11], and aircrafts [12] to gain a structure design with the best mechanical performance. However, the structure designs obtained by topology optimization are prone to be geometrically complex such as hollow structures and porous structures, which can hardly be fabricated using traditional machining. A compromising solution is that we consider the structural topology optimization problems with manufacturing constraints [13], which naturally sacrifices the mechanical performance of the optimized designs at some level.

Over the last two decades, additive manufacturing (AM) [14] also called 3D printing thrives. Unlike the traditional

*e-mail: JH.Zhu_FEA@nwpu.edu.cn machining methods, AM forms parts layer by layer through the joining of liquid, powder, or sheet materials. There are several AM technologies [15] including stereolithography, selective laser melting (SLM), three dimensional printing (3DP), and fused deposition modeling (FDM). Benefitting from its characteristics of manufacturing, AM is able to fabricate structure designs which are topologically complex, produce individualized products, and accelerate the process of manufacturing parts for no tooling needed at all. A lot of valuable researches has been done using AM technology recently. Castilho et al. [16] have explored to produce porous scaffolds for tissue engineering by means of a powder-based 3D printer. Moon et al. [17] investigate small and deployable UAVs wing designs using Objet 350 3D printer. Nevertheless the applications of stereolithography technology still are producing appearance models and durable concept models [18]. Considering the great need for massive topology optimized structure designs validation and the huge time and money cost for metal parts testing, here we propose to validate the topology optimized designs using prototype parts made by stereolithography.

In this study, we aim to develop an experimental approach to efficiently and quickly measure the mechanical performance of the structures designed using topology optimization. Taking the advantages of topology optimization and stereolithography, we can obtain an optimal structure design using topology 


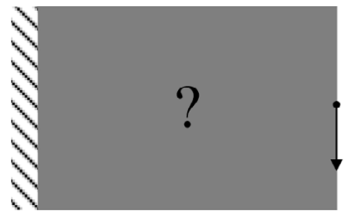

(a)

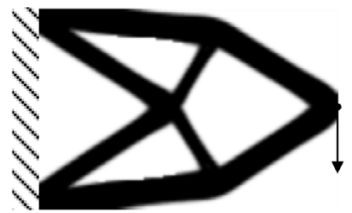

(b)
Figure 1. (a) Initial finite element model and (b) the optimal material layout.

optimization, fabricate it quickly and conveniently through stereolithography, and conduct a test to investigate the mechanical behavior of the design. So far we have developed a quick experimentally structure testing procedure. The finite element method is used to analyze the structure responses, and the consistent results of the numerical simulations and structure experiments prove the validity of the proposed methodology. Thus this new structure testing approach will not only provide a rapid access to topology optimized structures testifying, but also cut the turnaround time of structure design significantly.

\section{Basic formulation of topology optimization}

Topology optimization is used to gain the best material distribution within a design domain by seeking where to put the material (solid) and where not to (void) as shown in Figure 1. The topology of a structure can be represented by the density at different locations within the domain. The density is usually defined as a design variable and can assume 0 or 1 with 0 representing void and 1 representing the solid. The optimization method used in this work iteratively optimizes the elemental densities in the design domain to minimize the compliance (maximize the stiffness) of the final structure while satisfying the volume constraint. The mathematical form of the optimization model can be expressed as:

$$
\left.\begin{array}{rl}
\underset{\mathbf{x}}{\operatorname{minimize}}: & C(\mathbf{x}, \mathbf{u})=\frac{1}{2} \mathbf{u}^{\mathrm{T}} \mathbf{K u} \\
\text { subject to } & : \mathbf{K u}=\mathbf{f} \\
& : V(\mathbf{x})=\int_{\Omega} \mathbf{x} d \Omega \leq V_{s}
\end{array}\right\}
$$

Where $C(\mathbf{x})$ is the compliance, $\mathbf{x}$ is the density vector. $\mathbf{K}$ is the global stiffness matrix, $\Omega$ is the design domain. $\mathbf{f}$ and $\mathbf{u}$ are the global load vector and global displacement vector, respectfully; $V_{s}$ is the volume constraint. Employing the SIMP method, the problem is relaxed for density to have any value between 0 and 1 with small lower bound of $x_{\min }=0.001$ to avoid singularities during calculating for equilibrium. Also, a material interpolation scheme for local stiffness is defined. Thus, following is added in the problem.

$$
\begin{gathered}
0<x_{\min } \leq x_{i} \leq 1 \\
E(x)=x^{p} E_{0}
\end{gathered}
$$

Where $p$ is the penalization factor and $E_{0}$ is the Young's modulus of the material in the solid phase.

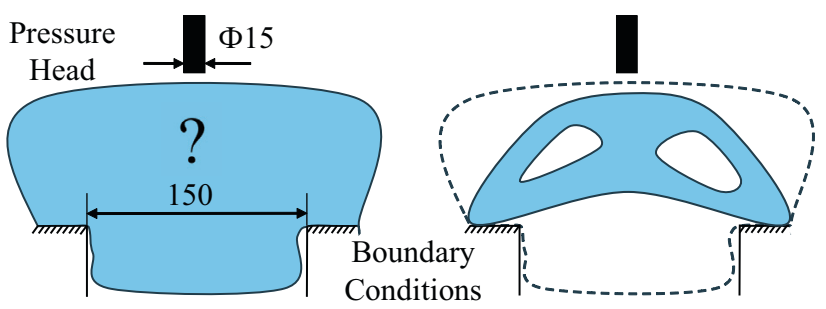

Figure 2. Illustration of the requirements of the structure competition.
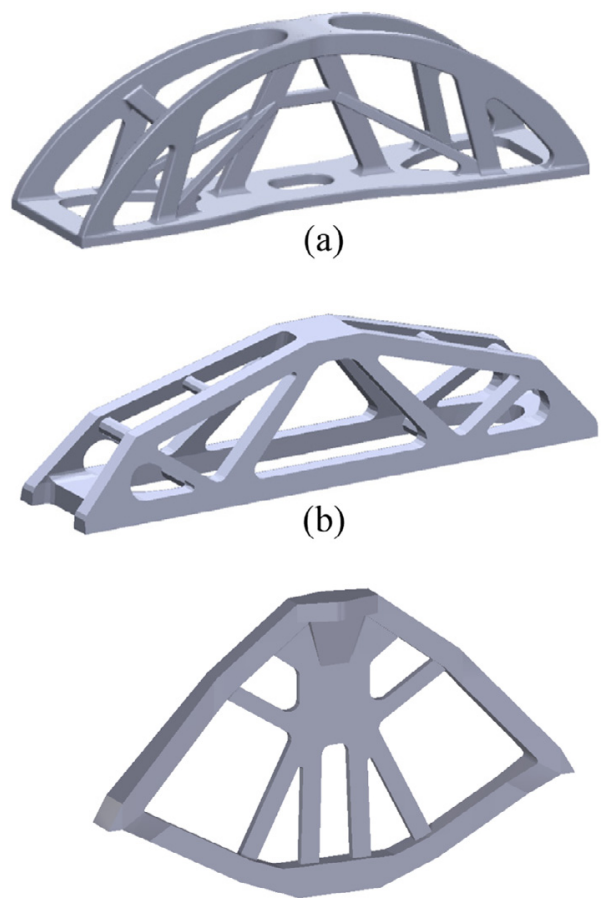

(c)

Figure 3. 3D beam design (a), (b) and (c) to the problem.

\section{Structure design}

\subsection{A 3d beam design problem}

In buildings, machineries, and bridge constructions, beams are one kind of regular parts highly used. A good beam design can not only guarantee the performance of the structure, but also satisfy mass constraint on the purpose of saving the material. So we set a 3D beam design problem as the topic of this structure design competition. Figure 2 shows the specific requirements of the problem. Between a $150 \mathrm{~mm}$ gap of two supporting columns, one needs to design a beam to withstand a compression force applied on the central area of the top face by a $15-\mathrm{mm}$-diameter pressure head and ensure the design satisfying the volume constraint of $30 \mathrm{~cm}^{3}$.

\subsection{Several beam designs given by contestants}

Figure $3 \mathrm{c}$ as an example to illustrates the design process briefly. At first we simplify this structure design problem as 


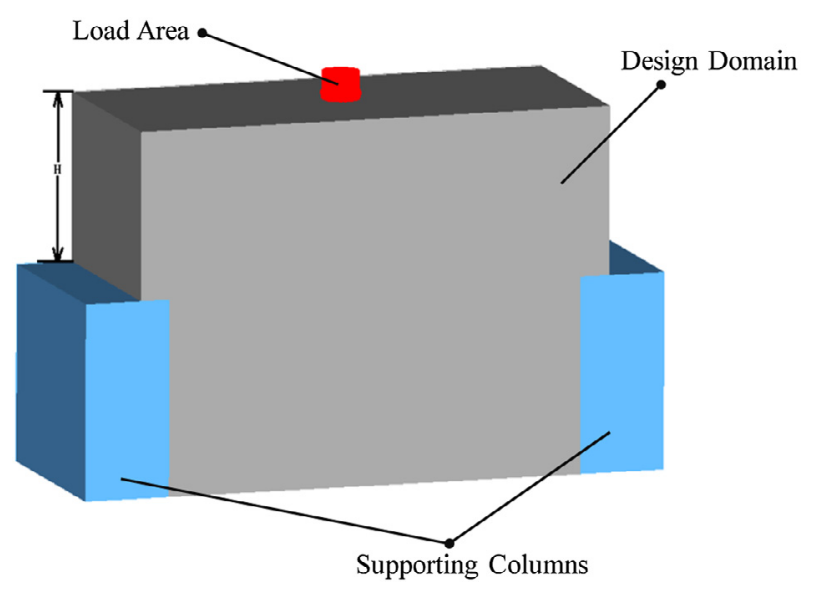

Figure 4. The minimum compliance topology optimization problem.

Table 1. The mechanical properties of photo-polymer DSM Somos 14120.

\begin{tabular}{llc}
\hline ASTM method & \multicolumn{1}{c}{ Description } & White 14120 \\
\hline D638M & Tensile strength & $45.7 \mathrm{MPa}$ \\
& Elongation at break & $7.9 \%$ \\
& Elongation at yield & $3.5 \%$ \\
& Modulus of elasticity & $2460 \mathrm{MPa}$ \\
& Poisson's ratio & 0.23 \\
\hline
\end{tabular}

a minimum compliance topology optimization problem and gain a minimum compliance solution, then reconstruct the design based on the optimal material layout obtained. The details are shown in the following steps.

\subsubsection{Step one}

Given the influence of initial domain size on the topology optimization solution, we choose height as the variation and investigate how it influences the optimal material layout as shown in Figure 4. In fact, other dimensional sizes of the design domain also have such influence, but we can eliminate that influence by giving adequate size along that orientations. The material properties are listed in Table 1, a total compression force of $1000 \mathrm{~N}$ is considered as the external load.

Then, topology optimization problems with different heights of design domain are solved, the relation between the compliance of the optimal material layout and the height of the design domain is shown in Figure 5.

From Figure 5 we can conclude that the optimal height for the initial design domain is $60 \mathrm{~mm}$, and the corresponding compliance is $0.276 \mathrm{~J}$. The optimal material layouts for the presented minimum compliance topology optimization problem are shown in Figure 6.

\subsubsection{Step two}

According to the optimal material layout obtained in the first step, the design is reconstructed (Figure 7), and numerically analyzed under the condition of applying $1000 \mathrm{~N}$ compression force using finite element method.

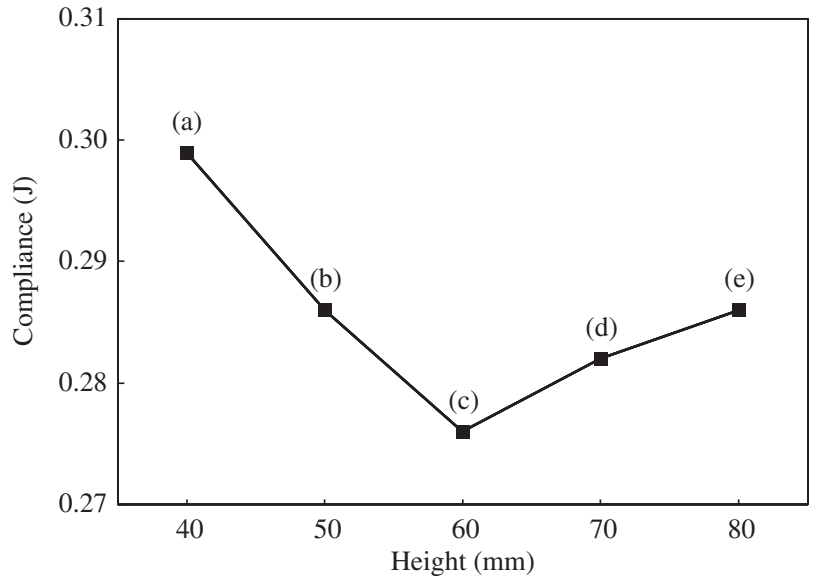

Figure 5. Compliance versus height.

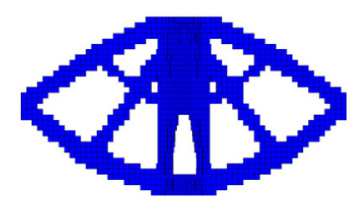

(a)

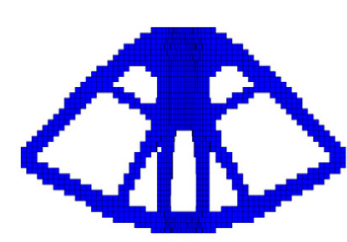

(c)

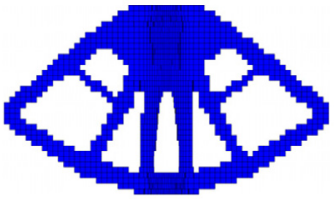

(b)

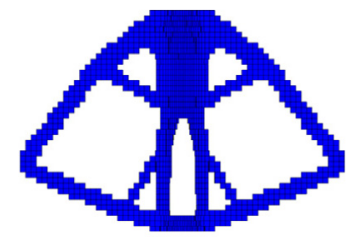

(d)

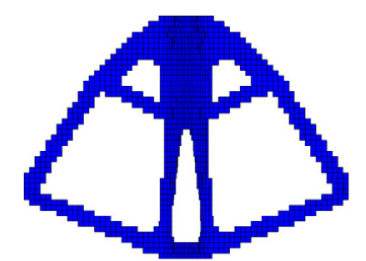

(e)

Figure 6. (a), (b), (c), (d) and (e) are the optimal material layouts for minimum compliance problems with different domain heights as in Figure 5

As shown in Figure 8, the reconstructed design well presents the load-carrying path of the topology optimized design, for the high-level similarity of the distribution of von Mises stress in two structures, and its compliance is $0.282 \mathrm{~J}$ which increases less than $2.2 \%$ than the optimized design. After the above two steps, a structure design with the maximum stiffness performance is obtained to this structure design problem.

\section{3D printing method and material properties}

\subsection{D printing method}

Stereolithography is one kind of liquid based 3D printing technology which processes with ultraviolet photo-curable 


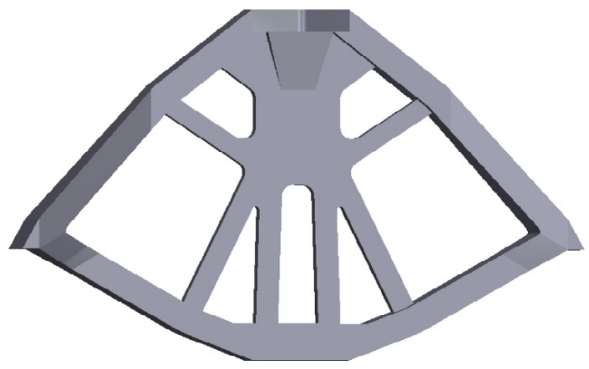

Figure 7. The reconstructed design.

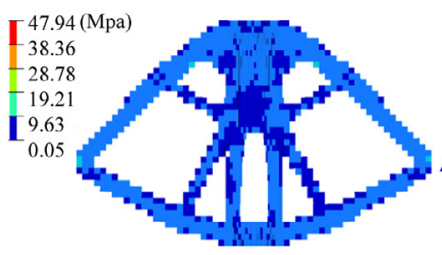

(a)

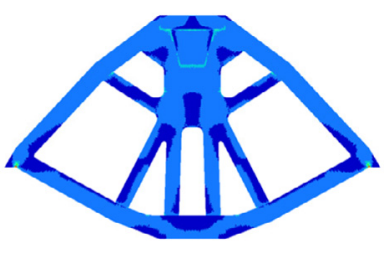

(b)
Figure 8. (a) von Mises stress in the optimized design and in (b) reconstructed design.

resin. Due to its capability of rapid manufacturing and the ingredient's characteristics, stereolithography has become an ideal method to produce dies of injection molding and casting. Here we choose stereolithography technology to fabricate all the structure designs for its high time efficiency and low fabrication cost. The 3D printer we use is SPS350B, which is invented by Shaanxi Hengtong Intelligent Machine Co., Ltd (SHIM) of China.

The process of stereolithography includes: modeling a solid part in computer with CAD software; converting the CAD file into Standardized Triangular Language (STL) file format to create volumetric mesh and the support structure; Slicing the volumetric-meshed model layer by layer and generate SLC file format; exporting the SLC file to computer of SLA; building the structure by curing the ultraviolet photocurable resin layer by layer using a ultraviolet ray; removing the support structure to get the design part; post-curing the part in an ultraviolet oven [19].

The material used in this experiment is DSM Somos 14120 , which is a low viscosity about $240 \mathrm{cps}$ at $30^{\circ} \mathrm{C}$ liquid photopolymer that produces strong, tough, water-resistant parts. The specific information about this material is given in Table 1.

\subsection{Mechanical properties of cured resin material}

Various process parameters affect the stereolithography process part quality characteristics, among which build parameters such as layer thickness, manufacturing orientation, hatch spacing have much influence according to the research of Raju et al. [20]. The research of Salmoria et al. [21] on somos 7110 resin reveals green samples fabricated by stereolithography process have layer ordering microstructures. Using the prototype parts produced by stereolithography to verify the mechanical performance of isotropic-material structure designs, it is
Table 2. Parameter levels for the experiment.

\begin{tabular}{lccc}
\hline Variable & Level 1 & Level 2 & Level 3 \\
\hline Manufacture orientations & $0^{\circ}$ & $45^{\circ}$ & $90^{\circ}$ \\
\hline
\end{tabular}

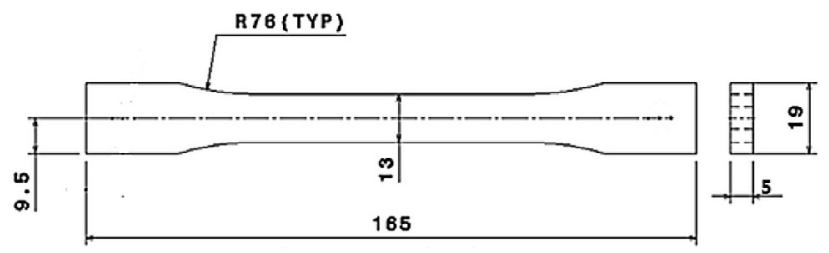

Figure 9. Tensile test specimen.

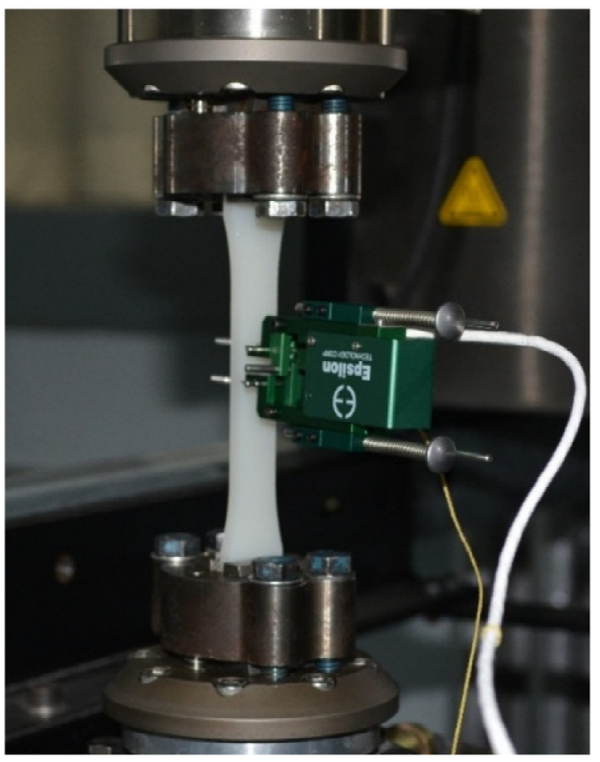

Figure 10. The setup of the standard tension test.

vital for us to investigate the degree of anisotropy caused by the nonuniformity existed in the stereolithography produced samples.

Fifteen tension specimens of five replications as per the ASTM standards (ASTM D638) are fabricated using DSM Somos 14120 resin in SLA SPS350B machine. The only variable between the three groups of specimens is manufacturing orientation, three levels are picked as show in Table 2 (Figures 9-11).

The elastic stiffness and yield strength of stereolithography produced samples of different manufacturing orientations are represented by the elastic modulus and yield stress shown in Figure 12.

From Figure 12, we can conclude that the anisotropy exists between the stiffness of different manufacture orientations is about $418 \mathrm{MPa}$, which is around $13 \%$ with respect to the stiffness of $90^{\circ}$ manufacture orientation and the anisotropy exists between the yield strength of different manufacture orientations is about $21 \mathrm{MPa}$, which is around 34\% with respect to the yield strength of $90^{\circ}$ manufacture orientation. Regardless of the slight anisotropy caused by the stereolithography 


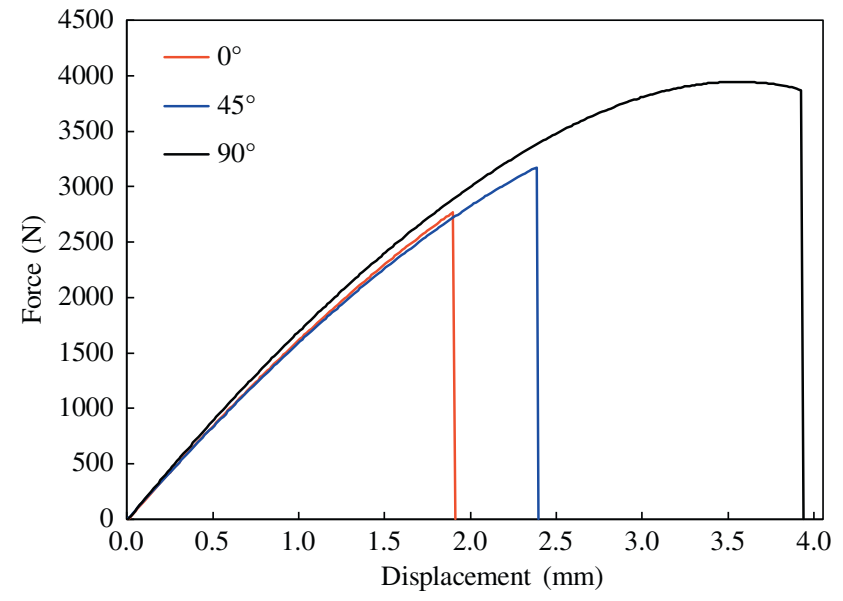

Figure 11. The load-displacement curves of specimens.

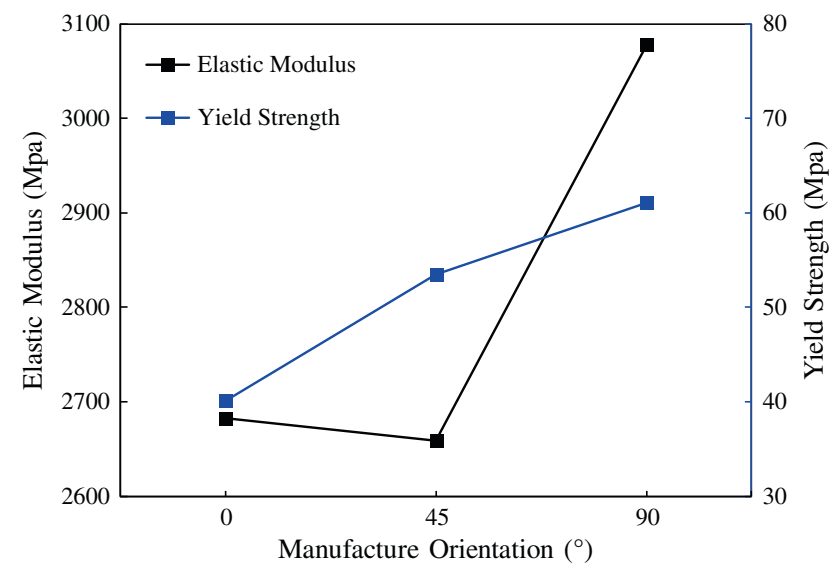

Figure 12. The elastic modulus and yield strength of different manufacture orientations.

process, we consider the material isotropic and the isotropicmaterial structure designs are tested using this stereolithography produced material.

\section{Experiment}

\subsection{Structure test procedure}

The static structure tests were carried out at a feed rate of $3.6 \mathrm{~mm} / \mathrm{min}$ using Testresources Testing System. All the structures were tested under the same conditions and experimental parameters. The test procedures were as follows:

1. Set the parameters of the experiment.

2. Place the structure on the test platform.

3. Lower the pressure head towards upper surface of the structure such that the structure is firmly held.

4. Preset zero the load and the displacement on the measuring scale and carry out the test.

5. Record the load applied on the structure and the displacement of the pressure head simultaneously.

6. Continue the test until the structure fails.

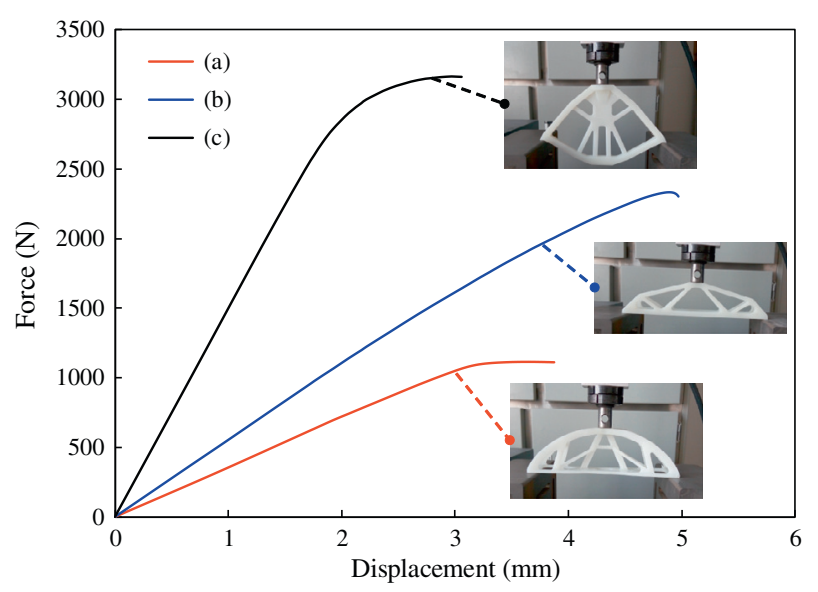

Figure 13. Load-displacement curves of the structures.

Table 3. Comparison of numerical simulations and experiment results.

\begin{tabular}{lccc}
\hline No. of the structure & (a) & (b) & (c) \\
\hline Compliance of reconstructed design (J) & 1.22 & 0.85 & 0.28 \\
Simulated displacement (mm) & 2.64 & 1.74 & 0.59 \\
Actual displacement (mm) & 2.83 & 1.80 & 0.68 \\
\hline
\end{tabular}

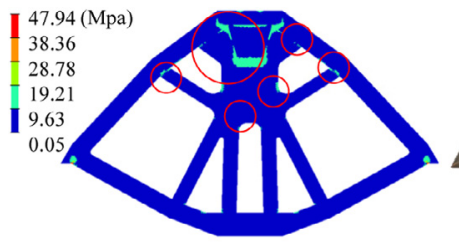

(a)

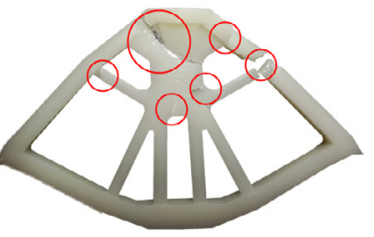

(b)
Figure 14. (a) The von Mises stress in design (c) and (b) the fragments of the tested parts.

At the end of the experiments, several load-displacement curves were generated for comparison. The results will be discussed in the next section in details.

\subsection{Results and discussions}

The test results of three proposed designs are shown in Figure 13. Take the load-displacement curve of structure (c) as example, It goes much steeper than two other curves, which indicates structure (c) has the better stiffness performance, and this conclusion can also be drawn from the numerical simulation results in Table 3 as the less compliance, the stiffer the design is. When compression force reaches $1000 \mathrm{~N}$, the actual displacement that structure (c) generates is $0.68 \mathrm{~mm}$ which is $15.25 \%$ percentage bigger than $0.59 \mathrm{~mm}$ of the numerical simulation, and for structure (a) and (b) the relative difference between the actual displacement and the simulated displacement are $7.20 \%$ and $3.45 \%$, respectively. The consistent displacements mean that it is advisable for us to ignore the tiny anisotropy existed in the light-cured resin material from the stereolithography process. Moreover the places where structure 
(c) falls apart are very close to the places where the von Mises stress concentrates in the simulation result as presented in Figure 14. Thus from the high accordance between the numerical simulations and experiment results, we can draw the conclusion that the approach of quickly testing the mechanical performance of the structure designs using prototype parts proves valuable.

\section{Conclusions}

In this study, we established a quick structure testing approach for topology optimized designs using topology optimization and stereolithography technologies. Conclusions of this work are as follows:

- successful application of fabricating load-carrying structures using stereolithography;

- established a quickly structure testing approach to verify the mechanical performance of structures designed using topology optimization.

Acknowledgements. This work is supported by National Natural Science Foundation of China (90916027, 51275424, 11172236), the 111 Project (B07050), Science and Technology Research and development projects in Shaanxi Province (2014KJXX-37), the Fundamental Research Funds for the Central Universities (3102014JC02020505).

\section{References}

1. Bendsøe MP. 1988. Generating optimal topologies in structural design using a homogenization method. Comput. Methods Appl. Mech. Eng., 71(2), 197-224.

2. Suzuki K, Kikuchi N. 1991. A homogenization method for shape and topology optimization. Comput. Methods Appl. Mech. Eng., 93(3), 291-318.

3. Bendsøe MP. 1989. Optimal shape design as a material distribution problem. Struct. Optim., 1, 193-202.

4. Zhou M, Rozvany GIN. 1991. The COC algorithm, part II: topological, geometry and generalized shape optimization. Comput. Methods Appl. Mech. Eng., 89, 197-224.

5. Mlejnek HP. 1992. Some aspects of the genesis of structures. Struct Optim, 5, 64-69.
6. Xie YM, Steven GP. 1997. Basic evolutionary structural optimization. Springer: London.

7. Allaire G, Jouve F, Toader AM. 2004. Structural optimization using sensitivity analysis and a level-set method. J. Comput. Phys., 194(1), 363-393.

8. Deaton JD, Grandhi RV. 2013. A survey of structural and multidisciplinary continuum topology optimization: post 2000 . Struct. Multidisc. Optim., 49(1), 1-38.

9. Guo X, Cheng GD. 2010. Recent development in structural design and optimization. Acta Mech. Sin., 26(6), 807-823.

10. Yang RJ, Chahande AI. 1995. Automotive applications of topology optimization. Struct. Optim., 9, 245-249.

11. Stromberg LL, Beghini A, Baker WF, Paulino GH. 2011. Application of layout and topology optimization using pattern gradation for the conceptual design of buildings. Struct. Multidisc. Optim., 43, 165-180.

12. Zhu JH, Zhang WH, Xia L. 2015. Topology optimization in aircraft and aerospace structures design. Arch. Comput. Methods Eng., 1-28.

13. Zhu JH, Gu XJ, Zhang WH, Beckers P. 2013. Structural design of aircraft skin stretch-forming die using topology optimization. J. Comput. Appl. Math., 246, 278-288.

14. Gibson I, Rosen DW, Stucker B. 2010. Additive manufacturing technologies. Springer: New York.

15. Pham DT, Gault RS. 1998. A comparison of rapid prototyping technologies. Int. J. Mach. Tool. Manuf., 38, 1257-1287.

16. Castilho M, Pires I, Gouveia B, Rodrigues J. 2011. Structural evaluation of scaffolds prototypes produced by three-dimensional printing. Int. J. Adv. Manuf. Technol., 56, 561-569.

17. Moon SK, Tan YE, Hwang JH, Yoon YJ. 2014. Application of 3D printing technology for designing light-weight unmanned aerial vehicle wing structures. Int. J. Precis. Eng. Manuf. Green Tech., 1(3), 223-228.

18. Melchels FPW, Feijen J, Grijpma DW. 2010. A review on stereolithography and its applications in biomedical engineering. Biomaterials, 31(24), 6121-6130.

19. Kruth JP, Leu MC, Nakagawa T. 1998. Progress in additive manufacturing and rapid prototyping. CIRP Ann. Manuf. Techn., 47, 525-540.

20. Raju BS, Chandrashekar U, Drakshayani DN. 2013. Optimization studies on improving the strength characteristic for parts made of photosensitive polymer. J. Inst. Eng. India Ser. D, 94(1), 35-41.

21. Salmoria GV, Ahrens CH, Fredel M, Soldi V, Pires ATN. 2005. Stereolithography somos 7110 resin: mechanical behavior and fractography of parts post-cured by different methods. Polymer Testing, 24(2), 157-162.

Cite this article as: Zhu J-H, Yang K-K \& Zhang W-H: Backbone cup - a structure design competition based on topology optimization and 3D printing. Int. J. Simul. Multisci. Des. Optim., 2016, 7, A1. 